
allemande

52-1 | 2020

Le catastrophisme environnemental

\title{
L'« exterminisme » selon Rudolf Bahro, ou comment survivre à la catastrophe
}

Anne-Marie Pailhès

\section{(2) OpenEdition}

1 Journals

Édition électronique

URL : https://journals.openedition.org/allemagne/2207

DOI : 10.4000/allemagne.2207

ISSN : 2605-7913

Éditeur

Société d'études allemandes

Édition imprimée

Date de publication : 1 juillet 2020

Pagination : 33-47

ISSN : 0035-0974

\section{Référence électronique}

Anne-Marie Pailhès, "L'« exterminisme » selon Rudolf Bahro, ou comment survivre à la catastrophe », Revue d'Allemagne et des pays de langue allemande [En ligne], 52-1 | 2020, mis en ligne le 06 juillet 2021, consulté le 20 février 2022. URL : http://journals.openedition.org/allemagne/2207 ; DOI : https:// doi.org/10.4000/allemagne.2207 


\section{L'«exterminisme» selon Rudolf Bahro, ou comment survivre à la catastrophe}

\section{- Anne-Marie Pailhès *}

Plusieurs décennies avant l'apparition de la «collapsologie» ou science de la survie après l'effondrement (terme qui semble actuellement surtout circonscrit au domaine français $\left.{ }^{(1)}\right)$, le philosophe allemand Rudolf Bahro avait acclimaté à la langue allemande le terme anglais d'«exterminism» pour parler d' «Exterminismus»: à la dimension pacifiste qui avait présidé à son apparition en pleine Guerre froide au sein de la Nouvelle gauche britannique, il avait adjoint le sens de destruction inéluctable de la planète non seulement au moyen d'armes (nucléaires en particulier), mais aussi comme aboutissement d'un processus irréversible, inhérent à la civilisation occidentale. Une thématique aujourd'hui familière qui faisait écho en Allemagne aux écrits des premières figures de proue de l'écologie politique au début des années $1970^{(2)}$.

Après la publication de L'Alternative en RFA en 1977, la pensée du philosophe estallemand n'a plus franchi le Rhin, jugée trop ésotérique pour intéresser encore la gauche française et peu pertinente car elle prétendait dépasser l'antagonisme des blocs de la Guerre froide pour donner une vision globale, planétaire, du destin de l'humanité. Bien que Bahro ait vécu et écrit jusqu'en 1997, soit vingt ans après L’Alternative, la seconde partie de son œuvre est passée totalement inaperçue du public français et aucun de ses ouvrages ultérieurs n’a été traduit en français, ni d’ailleurs en anglais.

* Maître de conférences HDR, Université Paris Nanterre, CEREG.

1 Il s'agit en fait de l'étude de l'effondrement de la civilisation industrielle, popularisée par le biais de ce néologisme sous la plume de Pablo Servigne et Raphaël Stevens dans leur essai Comment tout peut s'effondrer. Petit manuel de collapsologie à l'usage des générations présentes (Paris, Seuil, 2015).

2 Voir à ce sujet l'analyse d'Olivier Hanse sur les ouvrages de Hubert Weinzierl, Herbert Gruhl et Erhard Eppler: Olivier Hanse, «Le catastrophisme environnemental de H. Weinzierl, H. Gruhl et E. Eppler entre projections apocalyptiques et tentatives de résoudre une 'crise de l'espoir'», Le texte et l'idée, $\mathrm{n}^{\circ} 32$ (2018), p. 99-125. Les ouvrages de Gruhl et Weinzierl figurent dans la bibliothèque de Bahro, et il cite Eppler à l'occasion. 
Nous verrons à quel moment et dans quel contexte le terme d'« exterminisme » apparaît chez lui, comment il l'a d'abord utilisé dans le cadre de son enseignement en RFA, puis comment il l’a imposé comme concept clé de sa pensée dans l'ouvrage Logik der Rettung en 1987. Cette présentation des aspects spécifiques de son œuvre repose entre autres sources sur un travail au sein des archives Grünes Gedächtnis de Berlin, qui conservent le fonds le plus important concernant le philosophe ${ }^{(3)}$.

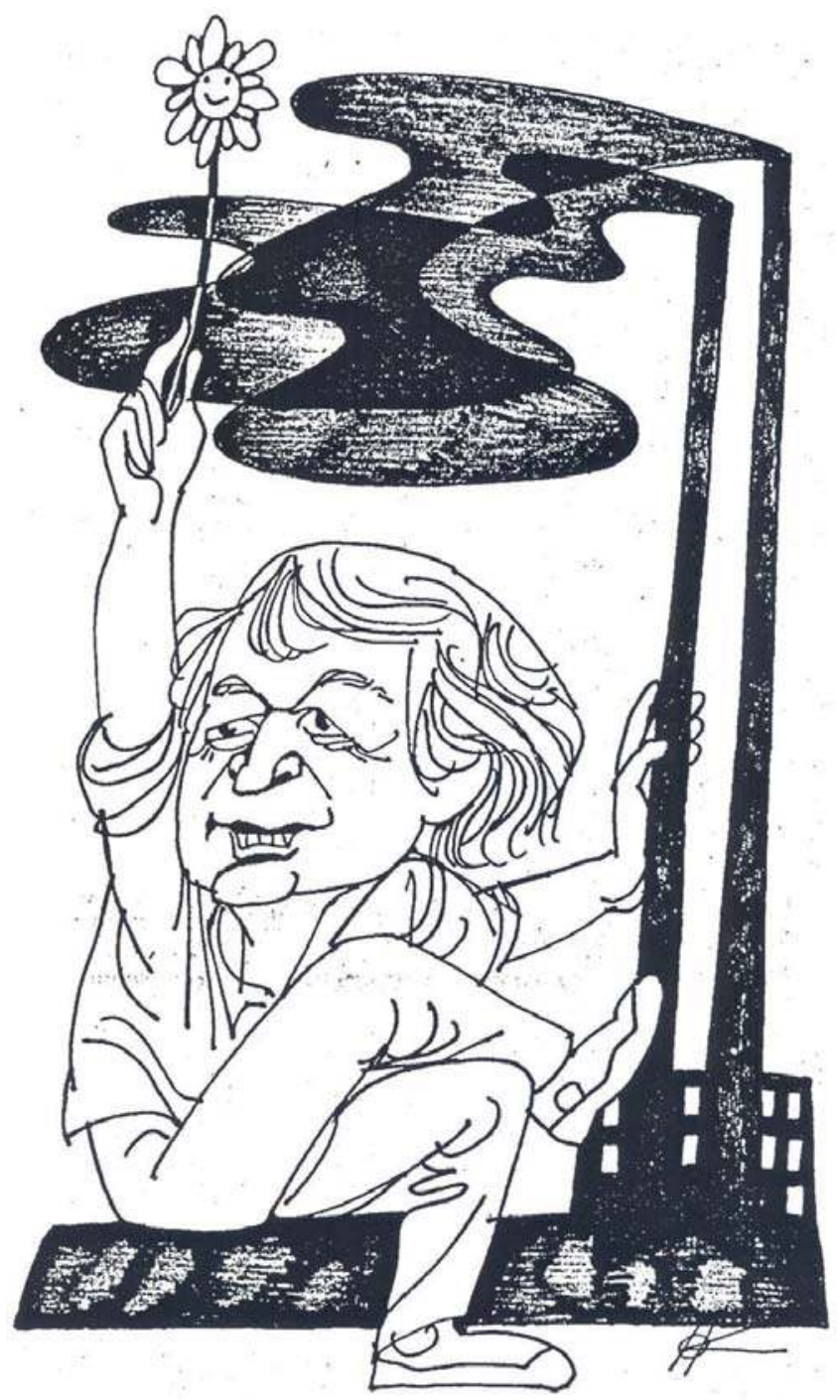

Ill. 1. Caricature de Rudolf Bahro

(C) Harald Kretzschmar, 1992

3 Il s'agit des archives de la Fondation Heinrich Böll, du parti des Verts. Elles abritent le fonds Bahro, constitué des archives données par sa veuve. D’autres documents sont disséminés à l'université Humboldt et dans la communauté de Pommritz, mais actuellement non mis en valeur. 


\section{$\mathrm{L}^{\prime}$ ' exterminisme» comme analyse du monde contemporain}

L'ouvrage qui avait rendu célèbre le dissident marxiste Rudolf Bahro, L'Alternative ${ }^{(4)}$, paru en 1977 en RFA, n'est pas prioritairement consacré à la thématique écologique; il a plutôt constitué à cette époque la Bible des réformateurs communistes des pays d'Europe de l'Est, et suscité l'espoir des partisans d'une «troisième voie» en Europe de l'Ouest.

Dans la première partie de L'Alternative, Bahro revient sur la genèse du socialisme et sur son évolution concrète en Union soviétique. La deuxième partie, «Die Anatomie des real existierenden Sozialismus » (L'anatomie du socialisme réellement existant), a suscité beaucoup d'intérêt chez ses lecteurs en raison de sa dimension critique: l'auteur y montrait comment la société de la RDA s'était éloignée de ses présupposés marxistes; il allait même jusqu'à assimiler le système économique qui y prévalait à un «ordre post-capitaliste» (nachkapitalistische Ordnung). Selon lui, la division du travail dans les sociétés dites socialistes ne diffère pas fondamentalement de celle des sociétés industrielles puisqu'il ne s'agit toujours pas de sociétés égalitaires, sans classes, mais que deux couches sociales distinctes y coexistent (il n'emploie pas le terme de classe): d'une part les décideurs responsables, d'autre part les exécutants subalternes (il insiste alors sur la notion de «subalternité », Subalternität). À ses yeux, le rôle dirigeant de la classe ouvrière en RDA est un mythe, l'appareil ne règne pas pour elle mais sur elle.

La troisième partie, «Zur Strategie einer kommunistischen Alternative» (Stratégie d'une alternative communiste), a été beaucoup moins abondamment commentée en raison de son caractère utopique qui l'éloignait de l'analyse du «socialisme réellement existant», alors qu'elle contient in nucleo la suite de son œuvre. Le philosophe y expose les principes économiques qui devraient être selon lui ceux du socialisme pour permettre l'évolution vers le communisme, le «royaume de la liberté» (Reich der Freiheit). Cette partie a été d'emblée qualifiée d'utopique et lui a valu une réputation tenace de "Spinner» (élucubrateur) ${ }^{(5)}$ dans des milieux intellectuels encore largement dominés par la doxa marxiste - Bahro tirant, quant à lui, plutôt son inspiration des socialistes utopistes français ${ }^{(6)}$. Rétrospectivement, on y trouve cependant des éléments déterminants de sa pensée ultérieure d'écologiste radical, qui seront en particulier développés

4 Rudolf Bahro, Die Alternative. Zur Kritik des real existierenden Sozialismus, Cologne/Francfortsur-le-Main, Bund-Verlag, 1979, p. 141-296. Trad. fr. L'alternative, Paris, Stock, 1979 (traduit sous la direction de Patrick Charbonneau, avec la collaboration de Roger Chudeau, Alain Cozic et Sabine Fontvieille), p. 113-234.

5 Guntolf Herzberg et Kurt Seifert, Rudolf Bahro, Glaube an das Veränderbare. Eine Biographie, Berlin, Aufbau, 2005, p. 181.

6 Ses visions utopiques, dans L'Alternative, sont plutôt marquées par la lecture des théories présocialistes des penseurs français tels que Babeuf, Proudhon, Fourier, Saint-Simon ou Blanqui. En témoigne la présence dans les archives d'ouvrages, datant de la période vécue en RDA, comme ceux de Babeuf et Saint-Simon, publiés en RDA dans les années 1950, ou ceux qui portaient sur les philosophes français pré-marxistes. Henri de SaInt-Simon, Ausgewählte Texte, mit einem Vorw., Komm. und Anm. von Jean Dautry, Berlin, Rütten \& Loening, 1957, 227 p. (AGG: Réf: Pol 042-5 - Standort: Bahro/047); Joachim Höppner, Waltraud SeIdel-Höppner, Von Babeuf bis Blanqui: Französischer Sozialismus und Kommunismus vor Marx, t. 1 : Einführung, Leipzig, Reclam, 1975, 541 p. (AGG: Réf.: Pol 042-6/1 : Bahro/050), ou bien Reise nach Utopia: französische Utopien aus drei Jahrhunderten, éd. par Werner 
dans Logik der Rettung ${ }^{(7)}$ (Logique du sauvetage) en 1987. Mais il reste à cette époque encore attaché à l'idéal communiste, visant un dépassement de la division du travail et de la «subalternité», la modification des habitudes de production et l'arrêt de l'exploitation de la nature, une nouvelle définition des besoins, l'émancipation des femmes, la création de communautés pour décentraliser l'exercice du pouvoir.

Quand Rudolf Bahro est libéré de prison après plus de deux ans de détention (entre août 1977 et octobre 1979) pour publication illégale à l'Ouest d'un ouvrage subversif, et qu'il arrive en Allemagne de l'Ouest, il est classé parmi les «Eurocommunistes» prêts au «compromis historique ${ }^{(8)}$ auquel appelait Enrico Berlinguer, secrétaire général du parti communiste italien et chef de file des Eurocommunistes. Il est cependant très rapidement attiré par un nouveau parti en cours de formation, Die Grünen/Les Verts, en lequel il voit ce «compromis historique». Carl Amery, écrivain écologiste et l'un des fondateurs du parti Die Grünen, avait d'ailleurs déjà reconnu dans l'auteur de L'Alternative un "Vert secret» ${ }^{(9)}$. Bahro sera un membre influent de ce parti depuis sa fondation jusqu'en $1985^{(10)}$. C'est au cours de ces années décisives du début des années 1980 que se situe sa rencontre avec le concept, opérationnel à ses yeux, d'«exterminisme», dans le contexte de l'affrontement de deux blocs par intimidation militaire.

À cette époque, le stationnement des fusées SS20 sur le territoire de la RFA (après la double décision de l'OTAN allant en ce sens en 1979) est contesté lors de grandes manifestations. En RDA, le mouvement pacifiste prend de l'ampleur avec le slogan «Transformez vos épées en socs de charrues!». Dans le long entretien accordé à la New Left Review ${ }^{(11)}$, From Red to Green, Bahro a décrit par la suite quelle a été son évolution intellectuelle pendant cette période. Il y dit:

"J'ai cherché le fondement anthropologique de [cette crise] et j'ai essayé de déterminer si la voie européenne était une spécialisation accidentelle de l'espère humaine ou bien si c'était une spécialisation qui, comme cela arrive souvent au cours de l'évolution, mènera à la destruction de l'espèce si elle continue sans cesse » ${ }^{(12)}$.

Krauss, Berlin, Rütten \& Loening, 1964, 478 p. (AGG : Réf. : Lit 200-46 - Bahro/110). Selon BibliotheksDatenbankauszug des Archivs Grünes Gedächtnis.

7 Rudolf Baнro, Logik der Rettung: Wer kann die Apokalypse aufhalten? Ein Versuch über die Grundlagen ökologischer Politik, Stuttgart, Weitbrecht, 1987.

8 Cité dans Herzberg/Seifert, Rudolf Bahro, Glaube an das Veränderbare (note 5), p. 333-335.

9 R. BAнro, Logik der Rettung (note 7), p. 9. Bahro confirme là ce qu'il avait déjà dit dans une interview au Spiegel en 1979.

10 Silke Mende, "'Rot und grün - das geht zusammen': Rudolf Bahro», in: "Nicht rechts, nicht links, sondern vorn ». Eine Geschichte der Gründungsgrünen, Munich, Oldenbourg (Ordnungssysteme. Studien zur Ideengeschichte der Neuzeit, Bd. 33), 2011, p. 250-258.

11 From Red to Green: interviews with New Left Review / Rudolf Bahro. Transl. by Gus Fagan and Richard Hurst, Londres, Verso Editions, 1984, 238 p. Interviews réalisées en 1980, 1981 et 1983. À ce jour, cet ouvrage n'a été traduit ni en allemand ni en français, alors que l'entretien a, bien entendu, eu lieu en allemand.

12 Ibid., p. 213: "I have also looked into the anthropological basis for all this and tried to determine wether the European way is an accidental specialization in the human species or wether it is a specialization which, as so often happens in evolution, will lead to the destruction of the species if it continues unbroken.» 
S’il y précise son emploi personnel du terme, il ne renvoie cependant pas dans ce texte aux penseurs britanniques qui l'ont inspiré, considérant probablement que les lecteurs de la revue sont parfaitement au fait de ce courant de pensée alors prégnant.

Issu du latin et dérivé du verbe anglais «exterminate», le terme a en effet été introduit pour la première fois par l'historien britannique Edward Palmer Thompson dans son article «Notes on Exterminism, the Last Stage of Civilization", d'abord publié dans la New Left Review (121, May-June 1980), puis presque immédiatement traduit en allemand ${ }^{(13)}$ avant d'être réédité dans plusieurs ouvrages (et traduit en français en 1983) ${ }^{(14)}$. Comme Bahro, Thompson était un ancien communiste, devenu militant pacifiste anti-nucléaire:

«[il] participe à la rédaction de l'Appel pour un désarmement nucléaire européen, plaide pour une Europe dénucléarisée et s'inquiète de la diffusion de plus en plus rapide des centrales atomiques et de la croissance de l'industrie nucléaire. [...] Le mouvement né en Grande-Bretagne essaime rapidement dans toute l'Europe, donnant naissance à une vaste campagne pacifique et antinucléaire. Thompson s'y investit avec fougue dans la première moitié des années 1980: il rédige des dizaines d'articles pour la presse européenne et américaine, apparaît fréquemment à la télévision, et multiplie les conférences et les interviews en Angleterre comme à l'étranger. Il s'en prend aux deux blocs, ce qui lui vaut d'être dénoncé autant comme un agent de la CIA que de l'URSS, et exhorte ses contemporains à abandonner la logique de la guerre froide pour réinventer une société pacifiée » ${ }^{(15)}$.

Le titre de son essai faisait clairement écho au texte de Lénine sur L'impérialisme, stade suprême du capitalisme (1917). Cependant, le titre de Thompson semble insister sur l'absence d'issue à l'état du monde qu'il décrit comme celui des «moulins de Satan ». Thompson appelle à l'insurrection des populations: à ses yeux, «les divergences secondaires doivent s'effacer devant l'impératif de survie écologique de l'humanité ${ }^{(16)}$. Le terme qu'il a popularisé a cependant eu une existence limitée à cette courte période des années 1980, son emploi par Bahro dans son ouvrage de 1987 est sans doute l'un des plus tardifs, rendu possible par une réinterprétation plus large du terme ${ }^{(17)}$.

La bibliothèque de travail de Bahro contenait presque tous les ouvrages de Thompson parus entre 1976 et 1982, en allemand ou en anglais ${ }^{(18)}$. Il transcrit le terme anglais de Thompson par «Exterminismus», un néologisme qui rencontre un écho

13 «Exterminismus als letztes Stadium der Zivilisation», Befreiung, Zeitschrift für Politik und Wissenschaft, $\mathrm{n}^{\circ} 19 / 20$ (1980), p. 12-45.

14 Edward Thompson, L'exterminisme. Armement nucléaire et pacifisme, Paris, PUF, 1983, à l'initiative d'Étienne Balibar, avec des réponses et commentaires de Noam Chomsky, Rudolf Bahro et Raymond Williams.

15 François Jarrige, E. P. Thompson, une vie de combat, La vie des idées, 6 janvier 2015, laviedesidees. fr/E-P-Thompson-une-vie-de-combat.html\#nh32, consulté le 29 février 2020.

16 Cité in: ibid.

17 «Exterminismus», in: Ulrich Albrecht et Helmut Volger, Lexikon der Internationalen Politik, Berlin, de Gruyter, 2019, p. 138-139.

18 Edward P. Thомpson, William Morris, 1976 - Edward P. Thompson, Das Elend der Theorie, 1980 Edward P. Thомpson, Plebejische Kultur und moralische Ökonomie, 1980 - Edward P. Thомpson, Writing by candlelight, 1980 - Edward P. Thompson, Beyond the cold war, 1982. Ouvrages présents sur la liste de la bibliothèque personnelle de Bahro, ouvrages qui ont tous été donnés au fonds Bahro des archives Grünes Gedächtnis. 
particulier car il renvoie à la Shoah, à Auschwitz, mais aussi aux propos du député Gustav Heinemann qui avait refusé en 1958 de désigner la bombe atomique comme une «arme» habituelle et l'avait qualifiée en allemand de Ungeziefervertilgungsmittel, c'est-à-dire de «moyen d'extermination de la vermine», l'homme jouant dorénavant le rôle de la vermine ${ }^{(19)}$.

L'essai de Thompson s'inscrit dans le contexte de l'exacerbation de la Guerre froide par la course à l'armement et l'intimidation militaire, en particulier nucléaire. Lors de la parution de L'Alternative en 1978, l'historien britannique avait déjà salué l'ouvrage de Bahro dans The Guardian comme «l'analyse la plus substantielle, la plus objective et finalement la plus optimiste du socialisme réellement existant » ${ }^{(20)}$, mais les deux hommes ne se connaissaient pas encore personnellement. Quelques années plus tard, entre 1980 et 1983, les noms de Bahro et de Thompson sont souvent associés dans la revue Befreiung ${ }^{(21)}$ ou dans des publications collectives pacifistes, comme Für ein atomwaffenfreies Europa ${ }^{(22)}$. Ces textes témoignent de l'apparition (ou de la réactivation?) d'un espace européen pacifiste de communication, d'un échange intellectuel constant au sein d'une partie de la gauche européenne en passe de devenir écologiste. La Fondation Russell pour la paix s'était par ailleurs fortement engagée pour la libération de Bahro en $1978^{(23)}$, pendant sa captivité.

C'est au cours de ces années pacifistes que Bahro a assimilé le concept d'exterminisme à sa propre pensée pour le réutiliser en un sens écologiste. Les pacifistes souhaitaient clairement dépasser le raisonnement de la Guerre froide, qui était alors celui d'une partie de la gauche ouest-allemande qui ne critiquait la politique américaine que dans le but de justifier la stratégie soviétique. Pour le nouveau mouvement pacifiste, la politique de réarmement et de dissuasion nucléaire devait être rejetée conjointement par les populations de l'est et de l'ouest de l'Europe.

\section{Enseigner l'analyse de l'exterminisme}

En 1980, Rudolf Bahro se voit proposer un projet de recherche de trois ans à l'université de Brême, où il donne quelques cours magistraux à partir de $1981^{(24)}$. L'un d'entre eux est consacré à l'«exterminisme» et ses notes et documents de cours sont conservés dans le fonds Bahro des archives Grünes Gedächtnis.

19 Déclaration de Gustav Heinemann au Bundestag, 25 mars 1958.

20 Cité dans Herzberg/Seifert, Rudolf Bahro, Glaube an das Veränderbare (note 5), p. 273.

21 Ainsi, dans un numéro de la revue Befreiung - Zeitschrift für Politik und Wissenschaft, $n^{\circ} 4$ (1981), figurent un article d'Edward P. Thомpson («Megatote sind nicht zum Lachen» - «Des millions de morts: il n'y a pas de quoi rigoler») et un texte de BAHro qui répond à Thompson: «Überlegungen zu einem Neuansatz der Friedensbewegung Deutschland im Anschluß an E. P. Thompsons Aufsatz über Exterminismus als letztes Stadium der Zivilisation» («Réflexions sur un nouveau point de départ pour le mouvement pacifiste en Allemagne, à partir de l'essai d'E. P. Thompson sur l'exterminisme comme stade ultime de la civilisation»).

22 Edward P. Thompson, Ken Coates, Rudolf Bahro und Michael Vester, Für ein atomwaffenfreies Europa, hg. von westdeutschen Unterstützern des Aufrufes der Russell-Friedens-Stiftung, Berlin, 1981.

Herzberg/Seifert, Rudolf Bahro, Glaube an das Veränderbare (note 5), p. 216.

Ibid., p. 338. Les auteurs écrivent que le philosophe aurait finalement été rarement présent à Brême. 
À partir de ces documents, il est possible de reconstituer la formation d'une pensée parfois peu accessible, souvent poétique, aux accents religieux. Le dossier conservé permet de consulter un résumé d'une page du cours, deux feuilles distribuées aux étudiants (comprenant une problématisation de la notion et une bibliographie) et un exemplaire typographié de l'essai «Notes pour un cours sur 'Les dimensions de l'exterminisme et l'idée de l'émancipation générale' " ${ }^{(25)}$.

Dans From Red to Green, Bahro souligne le caractère complexe du concept dans lequel il distingue quant à lui quatre sens différents. Le premier est effectivement emprunté à E. P. Thomson et se réfère à la surenchère militaire qui peut mener à la guerre nucléaire. Quelques années plus tard, dans son ouvrage de 1987 intitulé Logik der Rettung, Bahro est revenu sur le rôle décisif du concept forgé par Thompson dans l'élaboration de sa propre pensée écologique. Dès la première partie consacrée expressément à l'exterminisme, il écrit:

«J'ai tout de suite ressenti que l'exterminisme ne s'appliquait pas seulement à la destruction militaire, à des inventions telles que la bombe à neutrons qui n'élimine que le vivant, mais en fait à toute la civilisation industrielle dans beaucoup de ses aspects, et pas seulement les aspects matériels, même si ces derniers sont les plus visibles» ${ }^{(26)}$.

Le deuxième sens évoqué est synonyme de famine de masse dans le tiers-monde, menant à l'extermination de la population. Le troisième est plus spécifiquement écologique: «Destruction de la nature comme résultat de la poursuite de l'industrialisme» ${ }^{(27)}$. Le quatrième est en fait celui sur lequel le philosophe pense qu'une action sera possible: «Un élément psychologique et anthropologique du pouvoir dans la psychologie humaine est exterministe» ${ }^{(28)}$.

Le texte du cours (14 pages) est un commentaire d'un tableau qu'il a élaboré pour éclairer sa vision globale de l'histoire d'une humanité qui va à sa fin, et des voies possibles pour échapper à la catastrophe. Par la suite, Bahro aura souvent recours à ce type de schéma à l'aspect rationnel et implacable pour expliciter son système de pensée, mais il ne reproduira que des parties de ce schéma complet. Ce tableau se présente comme une somme de toute sa théorie et donne un aperçu général sur la pensée foisonnante de l'auteur et ses références empruntées à des auteurs variés, des aires culturelles et des époques diverses ${ }^{(29)}$.

25 Notizen für eine Vorlesung über Dimensionen des Exterminismus und die Idee der allgemeinen Emanzipation, Berlin, Archiv Grünes Gedächtnis, fonds Bahro, dossier n 141 . Il s'agit de 14 pages typographiées, raturées, avec quelques ajouts manuscrits, et un schéma. Ce texte a été publié dans la revue Befreiung, ${ }^{\circ} 27$ (1983), p. 19-33, et repris dans Rudolf BAHro, Pfeiler zum anderen Ufer, numéro spécial de la revue Befreiung, Berlin, 1984, p. 184-199.

26 R. BAHRo, Logik der Rettung (note 7), p. 27 : «Ich empfand gleich, daß Exterminismus nicht nur auf militärischen Overkill, auf solche Erfindungen wie die Neutronenbombe, die nur Lebendiges vernichtet, paßt, sondern tatsächlich auf die Industriezivilisation insgesamt und in sehr vielen, nicht nur materiellen Aspekten, obgleich diese letztere zuerst ins Auge fallen. »

27 Ibid., p. 214: «Destruction of nature as a result of the continuation of industrialism.»

28 Ibid.: "A psychological and anthropological element of power in the human psychology is exterminist.»

29 Ce schéma est reproduit sur deux pages dans la revue Befreiung, mais ne comporte pas les annotations manuscrites de l'exemplaire conservé en archives. 


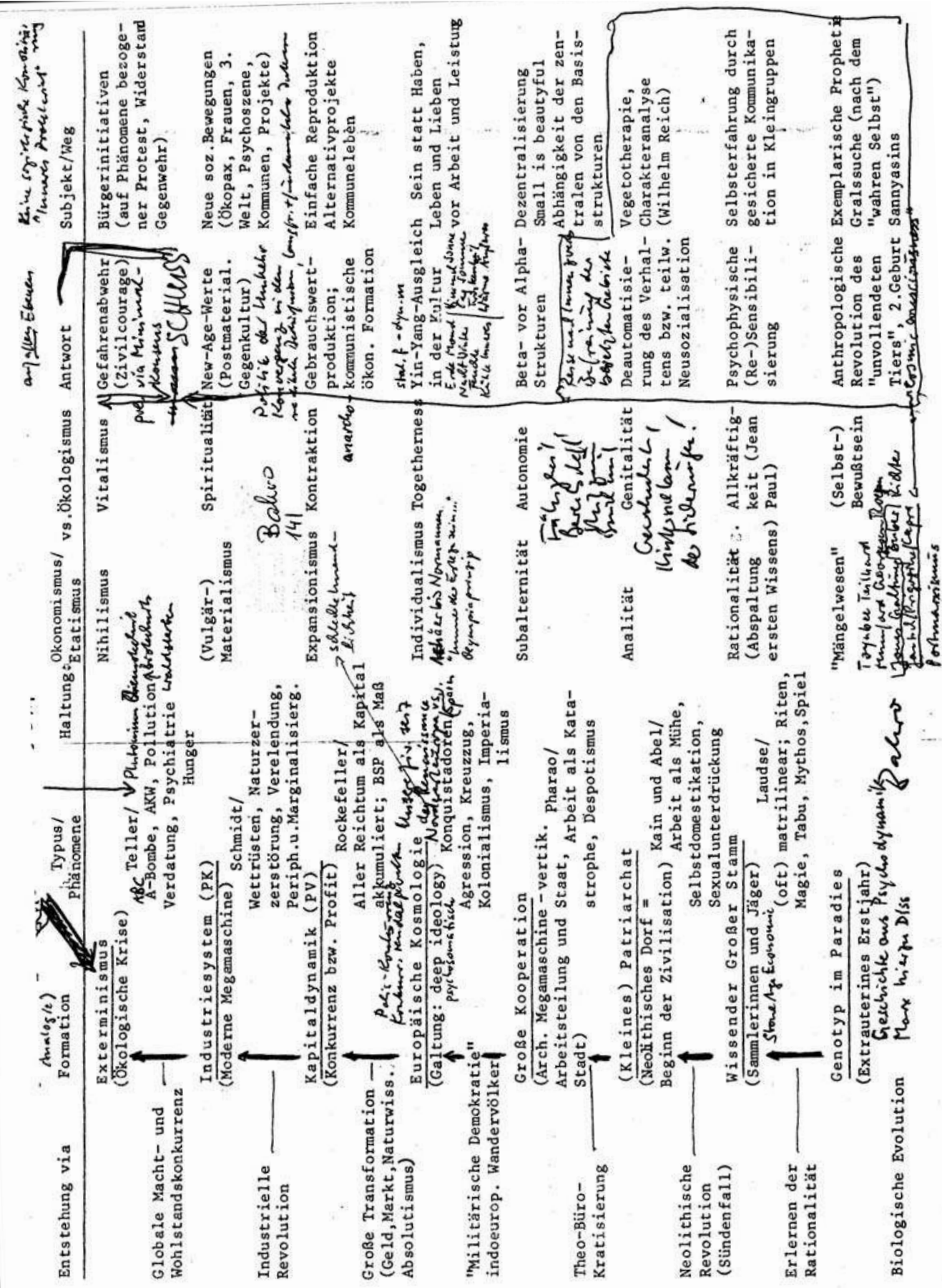

Ill. 2. Le schéma de l'exterminisme selon Rudolf Bahro, 1981 
D'un point de vue chronologique, le tableau se lit de bas en haut, soit à l'inverse de la logique occidentale de la lecture qui veut que l'on lise de haut en bas. Le choix de ce déroulement a un sens: il s'agit bien d'inverser l'analyse du monde, ce qui semble en être le début (= l'exterminisme) en étant la fin, l'apocalypse. Lire le tableau permet de suivre les étapes d'une généalogie des civilisations. Ce tableau comporte sept colonnes (chiffre magique récurrent) qui décrivent la marche inexorable de l'humanité vers sa fin, en huit étapes qui mènent du «Génotype du paradis» (situé tout en bas) à l'« Exterminisme» de la crise écologique, en passant par le "Grand Clan sage», le "(Petit) patriarchat ", la "Grande coopération», la "Cosmologie européenne », la «Dynamique du capital» et le «Système industriel». Les flèches qui vont de bas en haut indiquent la marche chronologique. Les huit étapes indiquées sont en fait inspirées des «formations sociales» reprises à la terminologie marxiste, au sens d'états de sociétés. La colonne située la plus à gauche explicite les différentes phases, qui sont aussi décrites dans des «sous-titres» entre parenthèses.

La troisième colonne porte l'indication «type/phénomène», elle donne pour chaque phase des exemples par lesquels elle se manifeste, en citant des concepts et des auteurs. Par exemple, la phase de «Grande coopération » est celle du «Pharaon», le travail étant alors perçu comme une catastrophe liée au despotisme. La phase du «Grand Clan» renvoie à Lao-Tseu, aux rites, aux tabous, au mythe, au jeu.

Si on se concentre sur la phase de l'exterminisme, on remarque qu'elle porte le soustitre de "crise écologique», caractérisé par les phénomènes suivants: bombe $\mathrm{ABC}$, centrales nucléaires, pollution, psychiatrie, famine, "mise en données» (Verdatung). Bahro a fait des ajouts manuscrits qui renvoient en particulier au contexte spécifique du début des années 1980: «Plutonium, technologie chimique, biotechnologie, dépérissement des forêts». Dans son texte, il date le début de l'étape exterministe de la Première Guerre mondiale qu'il compare ironiquement au vaillant petit tailleur des Frères Grimm qui tue «sept mouches d'un coup ».

La moitié de droite du tableau (colonnes 4 à 7) propose l'analyse des réactions à ces phases et suggère ce que serait la «voie» (Weg) de l'individu pour échapper à la conclusion exterministe. Il oppose ainsi le vitalisme écologique au nihilisme résultant de l'action de l'État et donne l'exemple d'actes de résistance individuels: défense face au danger, initiatives civiques qui apparaissent en réaction à un danger. Cependant, on voit clairement sur le schéma qu'il a entouré d'un trait toutes les caractéristiques anthropologiques des phases plus anciennes pour les amener par une flèche vers le haut du tableau. En effet, les réactions individuelles de type «initiative citoyenne» sont à ses yeux purement réactives et il serait selon lui bien plus efficace pour l'être humain de se reconnecter à ses structures psychiques archaïques : recherche du "véritable soi» comme le sannyasin du yoga, expérience de soi par le biais d'une communication en petits groupes, végétothérapie et analyse de caractère selon Wilhelm Reich.

Le texte quant à lui comporte tout d'abord sept pages d' "introduction" au propos, contenant souvent des digressions. Dans cette première partie, Bahro analyse les deux principaux concepts exposés, l'exterminisme et l'émancipation. Pour expliquer l'exterminisme au sens où il l'entend, c'est-à-dire un sens élargi qui ne se limite pas à l'extermination possible par les armes nucléaires, il se réfère souvent à l'historien britannique Arnold Toynbee, qu'il cite: «[...] la destruction qui a touché par le passé 
un certain nombre de civilisations n’a jamais été l'œuvre d'un agent extérieur mais s'est toujours déroulée à la façon d'un suicide» ${ }^{(30)}$.

Selon Bahro, l'être humain a emprunté une voie erronée vers l'émancipation, essentiellement portée par la «composante de pouvoir» de l'esprit humain. Au-dessus de l'entrée vers ce qu'il nomme «la mégamachine» (terme emprunté à Lewis Mumford) devrait se trouver l'inscription de Dante: "Vous qui entrez ici, abandonnez tout espoir.' Non seulement cette mégamachine ne vous libère pas, mais elle vous tue. Vous ne saisirez pas au passage le moindre pan du royaume céleste dans le court délai qui vous en sépare ${ }^{(31)}$. Au plus tard depuis l'époque moderne, le processus d'émancipation a, selon lui, un déroulement exterministe.

La seconde partie de cet essai décrit ce qui permettrait de sortir de la perspective exterministe. Les réactions à la situation, citées dans la $5^{\mathrm{e}}$ colonne, ne constituent pas selon lui une réponse pertinente: «Nous n’allons pas nous en sortir sur ce plan-là, nous ne ferons que nous rapprocher de la catastrophe en allant d'une campagne de protestation à l'autre ${ }^{(32)}$. Les réponses adaptées se situent selon lui dans la partie basse du tableau où il est question d'une synthèse religieuse au sens de "conscience cosmique", d'une seconde naissance au sens bouddhiste. Le philosophe cite ici des physiciens (comme Weizsäcker), mais aussi Frank Capra, le maître à penser du New Age, ou des figures telles que Bouddha et le Christ ou encore François d'Assise: elles ne devraient plus être des exceptions, mais entraîner des minorités assez importantes à quitter elles aussi le cercle de Mammon, du Karma et toutes les forces d'une pratique aliénée. Dans l'édition de la revue Befreiung, l'article est illustré par un portrait de Bodhidharma, le premier patriarche du zen qui a transmis son enseignement de méditation ${ }^{(33)}$.

Il conclut sur la nécessité d'avoir des «zones libérées de conscience» car le cerveau fait aussi partie de la «mégamachine ${ }^{(34)}$. Critiquant à nouveau la voie politique choisie par Die Grünen, il écrit : «L'accès à la société qui est à l'ordre du jour dans les pays métropolitains ne peut être atteint qu'à travers un 'voyage vers l'intérieur'.»

Son texte d'analyse philosophique et politique s'achève sur des considérations religieuses:

«Se mettre en route, individuellement, vers Dieu - pour (re-?)trouver le soi originel, pour faire l'expérience de l'unité - et partir collectivement pour le royaume de Dieu (qui porte de nombreux noms dans l'histoire), ce sont les deux faces d'une seule et même chose, elles n'existent finalement qu'ensemble. Salut!» ${ }^{(35)}$.

30 Notizen für eine Vorlesung (note 25), p. 3.

31 Ibid., p. 5.

32 Ibid., p. 9: «Ich gehe davon aus, daß wir auf dieser Ebene nicht durchkommen werden, sondern uns nur von einer Protestkampagne zur anderen immer näher an die Katastrophe heranführen lassen.»

33 Quelques mois après la publication de ce texte, Bahro a rendu visite au gourou Bhagwan aux ÉtatsUnis (en août 1983), un geste qui lui sera reproché par ceux qui ont vu en lui un «écolofachiste» à la pensée ésotérique.

34 Terme emprunté à Lewis Mumford, Mythos der Maschine, 1974.

35 Notizen für eine Vorlesung (note 25), p. 14: «Der individuelle Aufbruch zu Gott - zum (Wieder-?)Gewinn des ursprünglichen Selbst, zur Erfahrung der Einheit mit dem Ganzen - und der kollektive Aufbruch in das Reich Gottes (es hat historisch viele Namen) sind zwei Seiten ein und derselben Sache, die letzten Endes nur zusammen gehen. Salut!» 
Ce dernier mot est cité en français, il faut sans doute avoir à l'esprit l'étymologie du terme et y voir un appel au salut, tel qu'il sera formulé dans l'ouvrage Logik der Rettung.

Les documents du cours comprennent également une bibliographie classée selon cinq thématiques qui permettent de mieux cerner la progression de la pensée de Bahro:

1. Exterminisme - sur le plan militaire, origine du débat

2. Exterminisme - dimension écologique

3. Exterminisme - dimension économique mondiale

4. Exterminisme - dimension anthropologique

5. Tentatives de réponse.

Les étudiants sont invités à rédiger un compte rendu de 5 à 10 pages de l'un des ouvrages cités, dont la liste n'est ni exhaustive ni exclusive aux yeux de Bahro. Les ouvrages ainsi répertoriés portent le sceau d'un grand éclectisme mais ont tous été rédigés au $\mathrm{XX}^{\mathrm{e}}$ siècle (à l'exception de ceux de Lao-Tseu) et majoritairement en allemand, avec une forte minorité cependant d'ouvrages en anglais (de Thompson ou Galtung, par exemple) et une prise en compte d'ouvrages français (Jean-Jacques Servan-Schreiber, Roger Garaudy, André Gorz, Pierre Teilhard de Chardin, Alain Touraine). Sur une cinquantaine d'ouvrages, à peine deux ont été rédigés par des femmes.

\section{Quelle voie du salut après la catastrophe?}

Les biographes de Rudolf Bahro font remarquer au sujet de Logik der Rettung:

«Peu de gens auront lu ce livre jusqu'au bout, les idées de Bahro ont dû sembler trop spirituelles, trop religieuses, trop fantastiques, elles répondaient à la question: qui et qu'est-ce qui pourrait empêcher l'apocalypse, et comment pourraient-elles sauver l'humanité dans un État divin?» ${ }^{(36)}$.

L'ouvrage n'a été édité qu'une seule fois, à 4800 exemplaires (alors que L'Alternative avait été vendue à 80000 exemplaires six mois après sa publication $\left.{ }^{(37)}\right)$. Cette œuvre se situe exactement entre la publication de L'Alternative (1977) et le décès de Bahro (1997), et représente le point culminant de sa pensée spirituelle, après la critique marxiste du socialisme réellement existant et avant le retour à l'Est et l'expérimentation de diverses mises en pratique de ses idées. Certains y ont même vu une "Alternative Ouest», c'està-dire une analyse critique radicale de la société capitaliste. Il n'est pas indifférent que Logik der Rettung ait paru chez l'éditeur Weitbrecht, alors connu, entre autres, pour la publication d'ouvrages de science-fiction et celle des romans fantastiques de Michael Ende. Bahro a dédié cette œuvre à Ulrike Meinhof, à qui il vouait une grande admiration. Sa dédicace de 1987 prend la forme d'un poème dans lequel il dit ne pas croire à la thèse du suicide de la terroriste en prison; le dernier vers prend une dimension rétrospective et visionnaire supplémentaire, à deux ans de la fin de la RDA: «Überwindet die Mauern!» (Dépassez les murs!)

36 Guntolf Herzberg (éd.), Rudolf Bahro: Denker, Reformator, Homo politicus, Berlin, edition ost, 2007, p. 20: «Wenige werden das Buch bis zu Ende gelesen haben, zu spirituell, zu religiös, zu phantastisch müssen Bahros Vorstellungen wirken, wer und was die Apokalypse aufhalten könnte und wie sich die Menschheit in einen Gottesstaat hätte retten können. » 
Très opposé à la voie de l'éco-réalisme choisie par Die Grünen, il y affirme que «l'écosocialisme reste un exterminisme» ${ }^{(38)}$. L'ouvrage se scinde en quatre parties, dont les deux premières reprennent des thèmes connus. Il y décrit d'abord la situation de son époque («Koordinaten der Lage») en citant des exemples très concrets tirés du contexte allemand (Die Grünen, mais aussi l'homme politique du parti chrétiendémocrate, Kurt Biedenkopf).

La vision écologique qu'il esquisse dans Logik der Rettung prend des accents prophétiques: la seule alternative possible à ses yeux est une "conversion " (Umkehr) radicale de nature culturelle. Cette description d'une nécessaire "conversion» pourrait rappeler le principe de «conversion des cœurs» prêché dans le mouvement New Age. La position de Bahro est marquée par l'ambiguïté d'une attitude qui oscille entre la foi dans l'État et la foi en l'individu en tant qu'être sensible.

À la fin des années 1980, il fait partie de ceux qui suivent passionnément les changements en cours en URSS avec l'arrivée au pouvoir d'une nouvelle génération de communistes réformateurs en la personne de Michail Gorbatchev. Ce dernier semble incarner à ses yeux l'avènement du «prince vert» qu'il appelle de ses vœux dans la quatrième partie de Logik der Rettung.

Poussé par un enthousiasme qui peut sembler naïf à titre rétrospectif, Rudolf Bahro adresse, dès 1987, des lettres à Gorbatchev pour l'assurer de son entier soutien et le supplier de le laisser voyager en URSS ${ }^{(39)}$. La lettre du 6 mars 1987 est empreinte d'une foi réactivée en la vertu de l'État et du Parti; l'ancien fonctionnaire du SED, qui a officiellement tourné le dos au socialisme de type marxiste, semble ici repris de nostalgie à l'idée d'une nouvelle révolution possible par le haut. Cette lettre restée sans réponse est suivie d'une seconde missive, le 22 mars 1987 : elle accompagne cette fois-ci un paquet dans lequel Bahro envoie son œuvre au premier secrétaire du PCUS.

L'ouvrage développe tous les thèmes de la «tectonique de la perdition» qui mène la civilisation humaine à sa fin; les deux dernières parties sont consacrées à l'ordre nouveau (Ordine nuovo) qui devrait être instauré pour surmonter la catastrophe de l'effondrement: se retirant de la "mégamachine», l'être humain devrait trouver la conversion vers l'intérieur.

\section{Après le déluge, la mise en pratique des idées communautaires}

Le désastre de l'effondrement du communisme et la fin de la RDA en 1989 rendent possibles de nouvelles expérimentations. Après la catastrophe, la création de communautés permet à l'être humain de se raccrocher aux «canots de sauvetage» (Rettungsboote) que représenteront les communautés alternatives. Bahro vécut à partir de 1987 dans une communauté à Niederstadtfeld dans l'Eifel, qu'il décrivit lui-même comme un «nouveau cloître» ${ }^{(40)}$ et à laquelle il donna son empreinte décisive car il reste l'un des rares philosophes à avoir tenté de mettre ses idées politiques en pratique, à petite échelle.

38 R. BAнro, Logik der Rettung (note 7), p. 401: «Ökosozialismus bleibt Exterminismus.»

39 Archiv Grünes Gedächtnis, Berlin, fonds Rudolf Bahro, dossier n 1, lettre envoyée de Worms, 6 mars 1987.

40 Herzberg/Seifert, Rudolf Bahro, Glaube an das Veränderbare (note 5), p. 439. 
À l'origine, la Freie Lernwerkstatt für neue Lebens- uns Arbeitsformen e.V., «Libre atelier d'apprentissage de nouvelles formes de vie et de travail», est née de l'initiative de quelques coopérants (Entwicklungshelfer) de retour en République fédérale d'Allemagne qui s'étaient rencontrés en 1978 lors d'un séminaire sur les «alternatives dans l'agriculture, la médecine et la pédagogie» ${ }^{(41)}$. Rudolf Bahro fit la connaissance de Beatrice Ingermann, l'une des fondatrices, à une époque où le centre connaissait des difficultés et où le philosophe envisageait depuis plusieurs années de fonder une communauté; il était l'animateur au sein des Grünen, depuis 1983, d'un groupe de réflexion sur les communautés, la Bundesarbeitsgemeinschaft Kommune-Bewegung der Grünen (Groupe fédéral de travail des Verts sur le mouvement des communes). En 1984, il avait été l'initiateur de la rencontre de Burg Stettenfels: à cette occasion, il avait publié son texte Kommune wagen - 10 Thesen über die Richtung der sozialen Alternative ${ }^{(42)}$ (Oser la communauté, dix thèses sur l'orientation de l'alternative sociale); il y exposait sa conviction de la nécessité de fonder des communautés à l'image de celle des Bénédictins, seule solution pour faire face à l'évolution suicidaire de la société capitaliste occidentale « exterministe». À Niederstadtfeld, dans l'Eifel, il assura la partie théorique de l'animation du centre en organisant des séminaires et des conférences. Il donna au centre une orientation nettement éco-spirituelle, comme le montre ce programme trouvé en archives, intitulé «Ce que nous continuons à vouloir à Niederstadtfeld»:

«Le sujet principal en est LA VIE TOTALE - LA POLITIQUE TOTALE / Notre association renouvelle l'idée traditionnelle de l'unité de la recherche, de l'enseignement et de la pratique de vie, elle se comprend donc comme une sorte d'Autre université. La recherche inclut la recherche sur soi-même et les expériences et exercices adaptés de caractère individuel et collectif [...] La communauté s'organisera de telle façon que chacun puisse augmenter sa liberté intérieure. / L'objet principal de la recherche et de la recherche sur soi sera la mission de la vie, de la nature, de la divinité (quel que soit le nom qu'on lui donne) attribuée à l'être humain - jusque dans toutes les ramifications nécessaires pour la pratique et la théorie, celles des activités écologiques, sociales et politiques. Le but de la recherche est de comprendre à partir de notre pratique de vie communautaire les différentes tâches pratiques d'une voie de salut, d'en mettre les résultats en pratique et de les transmettre » ${ }^{(43)}$.

41 Document «Freie Lernwerkstatt für neue Lebens- und Arbeitsformen e.V. », 2 feuillets, Archiv Grünes Gedächtnis, Berlin, fonds Rudolf Bahro, dossier n 67.

42 Vorbereitungsreader zur Kommune-Bewegung, Burg Stettenfels bei Heilbronn, 21.-24. Juni 1984, éd. par Die Grünen, 1984, p. 5-7.

43 Archiv Grünes Gedächtnis, Berlin, fonds Rudolf Bahro, dossier n 67. Feuillet «Was wir in Niederstadtfeld wollen». Le feuillet porte à la main, tracée de l'écriture de Rudolf Bahro, la mention: «Das Programm unserer ökospirituellen und politischen Kommunität», «Entwurf von Beatrice und Rudolf, inzwischen angenommen» («Le programme de notre communauté éco-spirituelle et politique», «Esquisse de Beatrice et Rudolf, acceptée entre-temps»). Il est intéressant de noter qu'il utilise en allemand le terme de Kommunität qui renvoie à la communauté de type religieux. «Das Gesamtthema heißt also GANZHEITLICHES LEBEN - GANZHEITLICHE POLITIK. / Der Verein erneuert die überlieferte Idee der Einheit von Forschung, Lehre und Lebenspraxis, versteht sich so als eine Art Andere Universität. Forschung schließt Selbsterforschung und die geeigneten Experimente und Exerzitien individuellen und gemeinschaftlichen Charakters ein. [...] Hauptgegenstand der Forschung und Selbsterforschung wird der Auftrag des Lebens, der Natur, der Gottheit - oder wie immer wir das nennen wollen - an den Menschen sein - bis in alle vom Leben, von der Lehre und Praxis verlangten Verzweigungen ökologischer, sozialer und politischer Aktivitäten hinein.» 
On retrouve dans ces citations, qui pourraient être multipliées, une conception religieuse de l'écologie, qui se traduit par l'emploi en allemand de termes tels que Kommunität, Gottheit, Rettung.

Les programmes des années 1987, 1989 et 1990, conservés dans les archives, donnent un aperçu des activités pratiquées: en 1987, séminaire de deux jours sur «La crise écologique» par Rudolf Bahro, arrivé peu de temps auparavant, et d'autres formations proposées sur le carnaval et les masques, le travail avec les demandeurs d'asile, la taille et la greffe des arbres fruitiers, la fabrication de marionnettes, le vin biologique ${ }^{(44)}$. En 1989, Bahro prend plus de place dans le programme, il propose, avec d'autres intervenants, un séminaire «Spiritualité et politique», un autre sur «Le poids de l'endettement sur la dignité humaine», un troisième sur «L'état d'urgence écologique et la démocratie». Ces séminaires se distinguent de la mouvance New Age dans la mesure où le souci de l'écologie est toujours lié à sa dimension politique concrète. Les intervenants qu'il invite sont souvent des personnalités controversées aux yeux des partis traditionnels de gauche ou des Verts qu'il a quittés en 1985: par exemple, Franz Alt est plusieurs fois présent. Ce journaliste, chrétien engagé, membre du parti chrétien-démocrate, fut ensuite l'un des personnages en vue de l'ÖDP, Ökologisch-Demokratische Partei, un petit parti de la mouvance conservatrice de l'écologie. Bahro fait aussi venir le philosophe Jochen Kirchhoff pour un séminaire sur la musique.

La fin de la RDA et la réunification allemande le poussèrent alors à retourner à l'Est, un terrain idéal d'expérimentation sociale après la catastrophe de l'effondrement du système industriel de la RDA. Il y fonda la communauté Lebensgut Pommritz en 1991. Pendant 26 ans, elle posa la question des rapports entre l'État et l'individu pour affronter l'effondrement dans une petite structure de survie ${ }^{(45)}$.

Ce survol du parcours intellectuel de Rudolf Bahro au prisme de l'analyse de la catastrophe montre à quel point sa pensée élaborée dans les années 1970 et 1980 a conservé toute son actualité pour le $\mathrm{XXI}^{\mathrm{e}}$ siècle. La Guerre froide n'est plus de mise, mais la possibilité d'une issue "exterministe» de la civilisation est plus que jamais présente à nos yeux, non seulement sous la forme d'une guerre nucléaire mais aussi sous celle d'une destruction planétaire inhérente au processus de civilisation. Bahro fait partie de ceux qui n'ont pas seulement pensé cette destruction, mais qui ont élaboré une possible stratégie de survie par la force de conversion de l'esprit.

\section{Résumé}

Cette contribution montre comment le philosophe allemand Rudolf Bahro a emprunté la notion d'exterminisme à la critique du capitalisme par la Nouvelle gauche britannique (Edward P. Thompson) pour en faire un pivot de sa pensée écologique. Alors que dans L’Alternative, les jours meilleurs s'annonçaient dans une troisième partie utopique intitulée "Stratégie d'une alternative communiste», le départ de Bahro pour l'Ouest

44 Archiv Grünes Gedächtnis, Berlin, Fonds Rudolf Bahro, dossier n 67, Rundbrief, n 10 (1987).

45 Voir l'analyse de cette communauté dans Anne-Marie PAILHÈs, Communautés rurales en Allemagne de l'Est: vers une réunification des contre-cultures?, Paris, Presses de la Sorbonne Nouvelle, 2019, chapitre 6: «Lebensgut Pommritz, le fief octroyé aux Allemands de l’Est», p. 251-292. 
en 1979 entraîna un infléchissement de sa pensée lors de sa rencontre avec le parti des Verts en cours de fondation, dont il devint une figure représentative jusqu'en 1985. Dans Logik der Rettung (1987), l'utopie prit la forme d'un "Prince du tournant écologique» qui devrait sauver l'humanité du désastre écologique irréversible induit par le caractère fondamentalement "exterministe» de la civilisation. L'analyse est menée à partir des documents du fonds Bahro des archives Grünes Gedächtnis de Berlin.

\title{
Zusammenfassung
}

Dieser Beitrag zeigt, wie der deutsche Philosoph Rudolf Bahro den Begriff „Exterminismus" aus der Kapitalismus-Kritik durch die britische Neue Linke (E. P. Thompson) entlehnt hat, um ihn zu einem zentralen Element seines ökologischen Denkens $z u$ machen. Während in der Alternative im dritten Teil mit dem Titel „Strategie einer kommunistischen Alternative" bessere Zeiten angekündigt wurden, führte die Übersiedlung von Rudolf Bahro in den Westen 1979 zu einem Umdenken, als er die neu entstehende Partei Die Grünen kennenlernte; bis 1985 war er eine repräsentative Figur dieser Partei. In Logik der Rettung (1987) nahm die Utopie die Gestalt eines „Fürsten der ökologischen Wende" an, der die Menschheit vor der unaufhaltbaren Katastrophe retten sollte, die durch den grundlegend „exterministischen" Charakter der Zivilisation hervorgerufen würde. Die Analyse wird auf der Basis des Bahro-Nachlasses im Archiv Grünes Gedächtnis in Berlin geführt.

\begin{abstract}
This contribution shows how the German philosopher Rudolf Bahro borrowed the notion of exterminism from the critique of capitalism by the British New Left (Edward P. Thompson) to make it a pivot of his ecological thinking. While in The Alternative better days were anticipated in an utopian third part entitled "Strategy of a Communist Alternative", Bahro's departure for West Germany in 1979 led to a shift in his thinking after his meeting with the new Green Party, of which he became a representative figure until 1985. In Logik der Rettung (1987), utopia took the form of a "Prince of the ecological turn" who would save humanity from the irreversible ecological disaster induced by the fundamentally "exterministic" character of civilization. The analysis is based on documents from the Bahro collection of the Grünes Gedächtnis archives in Berlin.
\end{abstract}

